\title{
Does hormonal contraception prior to in vitro fertilization (IVF) negatively affect oocyte yields? - A pilot study
}

David H Barad ${ }^{1,2^{*}}$, Ann Kim ${ }^{1}$, Hala Kubba ${ }^{1}$, Andrea Weghofer ${ }^{3}$ and Norbert Gleicher ${ }^{1,2}$

\begin{abstract}
Background: As oral contraceptives (OCs) suppress anti-Müllerian hormone (AMH), and hormonal contraceptives $(\mathrm{HCs})$, likely, suppress functional ovarian reserve, this study was initiated to determine whether HC affect oocyte yields.

Methods: We investigated in a retrospective cohort study 43 oocyte donors in 71 in vitro fertilization (IVF) cycles, evaluating anti-Müllerian hormone (AMH) and oocyte yields as reflections of functional ovarian reserve (OR). In 25 IVF cycles egg donors were on HC within one month prior to IVF, and in 46 cycles they were not. Donors, based on their HCs, were further subdivided into 12 with less, and 13 with more androgenic progestins.

Results: While the three groups did not differ in age, age at menarche, BMI and AMH, oocyte yields among donors who utilized estrane- and gonane-derived (higher androgenic) HCs were lower 11.3 (95\% Cl 8.3 - 14.3) than either donors using no HCs 16.6 (95\% Cl 14.7 -18.4) ( $\mathrm{P}<0.05)$ or those using anti-androgenic HCs 19.0 (95\% Cl 12.2-25.8) $(\mathrm{P}<0.01)$. Significance was maintained after adjustments for the donor age and total FSH dose used in ovulation induction.

Conclusions: Even in young oocyte donors, high androgenic OC exposure appears to suppress functional ovarian reserve and oocyte yields. Since OCs are often routinely used in preparation for IVF, such practice may require reevaluation. Especially in women with diminished ovarian reserve OCs, and especially high androgenic progestin HCs, should, likely, be avoided.
\end{abstract}

Keywords: Oral contraceptives, Hormonal contraception, In vitro fertilization (IVF), Oocyte yield, Ovarian reserve, Androgens

\section{Background}

Because the response to ovarian stimulation, to a large degree, depends on choice of stimulation protocols, any definition of poor response in association with in vitro fertilization (IVF) has to be considered relative. Poor response is, however, universally seen as the production of fewer than expected oocytes and embryos [1].

While by many considered a standard protocol in IVF, long agonist stimulations, because of suppressive effects on ovaries, are often considered inappropriate for poor responders [2]. Instead, hormonal contraceptives (HCs)

\footnotetext{
* Correspondence: dbarad@thechr.com

${ }^{1}$ Center for Human Reproduction, New York, NY, USA

${ }^{2}$ Foundation for Reproductive Medicine, New York, NY, USA

Full list of author information is available at the end of the article
}

are regularly utilized in such patients in preparation for ovulation induction $[3,4]$. HCs are also used to reduce ovarian cyst formation [5] and to allow convenient scheduling of cycle starts [6,7]. Results, utilizing HCs, have, however, not always been favorable [8-10]. A recent Cochrane review found that pretreatment with combined oral contraceptives (OCs) led to poorer pregnancy outcomes [11].

The primary mode of action for HCs is thought to be suppression of pituitary gonadotropin secretion, secondarily leading to suppression of ovarian function $[12,13]$. So-called combined HCs are combinations of estrogen (generally ethinyl estradiol, EE) and a variety of progestins. Differences among $\mathrm{HCs}$, therefore, primarily reside with their progestational agents. Classical HCs have

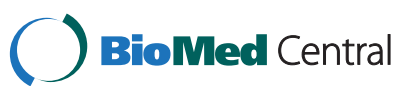


progestins, derived from estranes and gonanes, all to various degrees androgenic [14].

Synthetic progestins interact not only with progesterone receptors but also with other intranuclear steroid receptors [15]. Affinities for the various steroid receptors depend on the molecule from which a given synthetic progestin is derived [16]. In addition to progestational activity, steroid derivatives may also have androgenic, anti-androgenic, estrogenic, glucocorticoid or mineralocorticoid activities [16].

Consequently, one also has to conclude that different OCs, based on progestational content, should affect follicle maturation differently. Assuming this to be the case, different OCs could also be expected to affect functional ovarian reserve differently and, therefore, potentially oocyte yields.

Progestins may be classified into old progestins (norethisterone, levonorgestrel, gestodene) and new progestins (drospirenone, dienogest, trimegestone) [17]. Newer progestins are designed to be less androgenic. Like spironolactone, from which it derives, drospirenone functions as a competitive androgen antagonist $[18,19]$. Dienogest is a 19nortestosterone derivative but it differs significantly in structure from other nortestosterone derivatives [20]. An OC containing dienogest was recently approved for use in the United States [21].

$\mathrm{AMH}$ is considered a good marker of ovarian aging [22]. AMH levels generally correlate well with day $2 / 3$ FSH levels [23], and are good predictors of ovarian response to ovulation induction [24-26].

Suppressive effects of $\mathrm{HCs}$ on antral follicles and $\mathrm{AMH}$ levels have been reported. Women with polycystic ovarian syndrome (PCOS) who for 6 months took OCs, containing $35 \mathrm{ug}$ of $\mathrm{EE}$ and $2 \mathrm{mg}$ of cyproterone acetate, experienced significant decreases in antral follicles but no change in anti-Müllerian hormone (AMH) [27]. Cyproterone acetate is anti-androgenic but also suppresses gonadotropin secretion $[28,29]$.

Whether OC utilization suppresses AMH has remained controversial, with some studies supporting such an association [30,31], and others disagreeing [32,33]. This divergence suggests that different types of OCs, containing different progestins, and maybe different utilization length, could affect results.

Oocyte donors are selected to be healthy young women, without evidence of infertility and/or abnormal functional ovarian reserve. They often use OCs or other HCs. Especially in association with utilization of the socalled "contraceptive ring" (NuvaRing ${ }^{\mathrm{Tm}}$, Organon USA), which contains, and continuously releases, etonogestrel/ ethinyl estradiol, we noted in some of our egg donor applicants abnormally low age-specific AMH levels. Others noted diminished numbers of small follicles amongst women using contraceptive rings in comparison to those utilizing OCs, containing EE and levonorgestrel [34].
Considering all of these observations and reports, we in here presented study decided to formally investigate the effects of progestins on follicle maturation by determining how different $\mathrm{HCs}$ affect oocyte yields in young oocyte donors, stimulated with standard ovarian gonadotropin protocols.

\section{Methods}

\section{Population}

We retrospectivley reviewed the charts of 46 oocyte donors who underwent 71 IVF cycles. Amongst those, 43 underwent at least one donation cycle, 18 two cycles, 9 three cycles, and one donor 4 cycles. At least six months elapsed between oocyte donations for donors who contributed more than one cycle to the analysis. Since our donors are all young women some of them were using $\mathrm{HCs}$ as their method of contraception. The choice of type of $\mathrm{HC}$ was made by the donor's treating physicians outside of CHR. If used the $\mathrm{HC}$ used was recorded in each donor's medical record based on the donor's self report. Some donors changed their contraceptive status between donations and, thus, contributed in more than one category.

We routinely obtain AMH levels from donor candidates at their initial interview visit with a physician. Use of any hormonal contraceptives is allowed to continue until donor candidates are matched with a recipient, though some chose, on their own, to discontinue earlier. If more than six months elapse before a donor/recipient match, donor candidates are rescreened once matched, and before cycle start.

\section{Study qualifications}

Donors qualified for this study if at the time of IVF cycle start less than 100 days had elapsed from their last $\mathrm{AMH}$ assessment.

Oocyte yields were compared between 46 cycles where oocyte donors, prior to IVF cycle, were on no HCs, and 25 cycles where donors used HCs within one month of initiation of treatment. The latter group was further subdivided into 12 anti-androgenic (11 using contraceptives with drospirenone and 1 cyproterone acetate progestin), and 13 donors with the more androgenic estrane and gonane-derived progestins. Table 1 lists the various progestins used by women in this study.

Table 1 Progestins in HCs of oocyte donors

\begin{tabular}{lc}
\hline Progestin & Number of patients \\
\hline Drospirenone & 11 \\
Cyproterone Acetate & 1 \\
Norethindrone & 8 \\
Norgestimate & 4 \\
Norgestrel & 1 \\
\hline
\end{tabular}


Donors received the center's routine IVF cycle stimulation for oocyte donors, including down-regulation with luteal phase gonadotropin releasing hormone agonist, and gonadotropin stimulation with 150-300 IU daily of human menopausal gonadotropin (hMG). Products from different manufacturers were utilized, depending on patient preference and/or insurance mandates.

Serum levels of follicle stimulating hormone (FSH) and estradiol were evaluated on cycle day 2 to 3, using the Automated Chemo Luminescence System (ACS: 180, Bayer Health Care, Tarrytown, NY). Serum AMH levels were obtained through a commercially available assay, which involves an enzymatically amplified two-site immunoassay, DSL-10-14400 active MIS/AMH ELISA (Esoterix Endocrinology, Casabasas Hills, CA).

\section{Clinical outcomes}

Ovarian response was monitored by serial assessments of serum estradiol and transvaginal ultrasounds of ovarian follicular growth. Oocyte maturation was triggered with 10,000 IU of human chorionic gonadotrophin (hCG), when at least three dominant follicles had attained a size of 18-22 mm. Oocyte retrieval, guided by transvaginal ultrasound, was performed approximately 34 hours after hCG administration. Oocyte yields were registered in routine fashion by the embryology staff.

\section{Statistical analyses}

Baseline characteristics of the groups were tabulated as means and 95\% confidence intervals (CI) of the mean. Differences at baseline between groups were, when normally distributed, evaluated by one way analysis of variance, and by the Mann-Whitney U-test, when nonnormally distributed. A generalized linear model was used to adjust for the potential confounder of age and FSH dose, used in ovulation induction, and further adjusted for repeated cycles. A P-value $<0.05$ was considered significant. All analyses were carried out with SPSS software for Windows version 19.0 (SPSS Inc. Chicago, IL.)

\section{Ethical approval}

The study underwent expedited Institutional Review Board review, since it involved only analyses of anonymous medical records. At their initial consultation, all of the center's patients sign a consent, which allows use of their anonymous medical records for quality control and research purposes, as long as their medical records remain confidential and their identity protected. Both conditions were met. In addition, all staff with access to research data at our center, under Federal HIPAA rules, confirms their obligation to confidentiality in writing.

\section{Results and discussion}

For the whole study group mean age was $24.2 \pm 4.0$ years; mean AMH $4.4 \pm 2.9 \mathrm{ng} / \mathrm{mL}$; and mean oocyte yield was $15.6 \pm 7.7$. Time elapsed between last $\mathrm{AMH}$ and IVF cycle start was $8.9 \pm 3.6$ weeks.

There was no difference in age, age of menarche, BMI and $\mathrm{AMH}$ among the three groups (Table 2). Mean $\mathrm{AMH}$ was non-significantly higher without $\mathrm{HC}$ use but did not differ significantly between the three groups. This analysis was further limited by the fact that not all study subjects had AMH values available for analysis.

Oocyte yields among donors, who utilized more androgenic HCs, like estrane and gonane derived HCs, were lower 11.3 (95\% CI 8.3 - 14.3) than those of either donors using no HCs at all 16.6 (95\% CI 14.7 -18.4) (p < $0.05)$ or than those using anti-androgenic contraceptives 19.0 (95\% CI 12.2-25.8) ( $<$ 0.01) (Figure 1). Comparing androgenic $\mathrm{HCs}$ to the non-androgenic and no $\mathrm{HC}$ combined the omnibus test of significance was $(\mathrm{p}=0.018)$ after adjusting for donor age age and total gonadotropin dosage the significance was $(\mathrm{p}=0.03)$.

In the introduction section of this manuscript we laid out in detail why, depending on included progestational agent, HCs should be expected to affect functional ovarian reserve in different ways. Investigating young oocyte donors, this study presents support for this concept, suggesting that recent use of HCs affects response of healthy young women to ovulation induction, resulting in significantly different oocyte yields, depending on whether HCs were utilized or not, and depending on what HCs were used. HC-use before IVF, therefore, quite apparently reduces the response to ovarian stimulation, producing lower than expected oocyte numbers. HCs, therefore, under a recently summarized consensus definition of poor ovarian response, qualify as inducers of relatively poor ovarian response to stimulation [1].

Since our center, at most, transfers two embryos in donor/recipient cycles, a diminution in oocyte yields, as here reported, likely, will not affect immediate, freshcycle pregnancy rates. Since total available oocytes, and especially embryos, do, however, reflect cumulative pregnancy chances, it is reasonable to conclude that any diminution of oocyte yields will also negatively affect cumulative pregnancy chances. Especially for recipients, desirous of more than one child, this may be of relevance.

The utilization of HCs in women with less favorable functional ovarian reserve than young oocyte donors, as represented by many women under standard infertility treatments with use of autologous oocytes, who may produce only relatively small oocyte yields, can, however, also be expected to affect fresh-cycle pregnancy rates since in such cycles the transfer of larger embryo numbers may be indicated. 
Table 2 Donor characteristics and oocyte yields in reference to contraceptive use

\begin{tabular}{|c|c|c|c|c|c|c|c|c|c|}
\hline & \multicolumn{3}{|c|}{ No contraception } & \multicolumn{3}{|c|}{ Androgenic } & \multicolumn{3}{|c|}{ Anti-androgenic } \\
\hline & $\mathrm{N}$ & Mean & $95 \% \mathrm{Cl}$ & $\mathrm{N}$ & Mean & $95 \% \mathrm{Cl}$ & $\mathrm{N}$ & Mean & $95 \% \mathrm{Cl}$ \\
\hline Age & 46 & 25.0 & $24.0-26.1$ & 13 & 23.5 & $21.4-25.6$ & 12 & 24.0 & $20.3-27.7$ \\
\hline Menarche & 46 & 13.2 & $12.8-13.7$ & 13 & 12.8 & $12.1-13.5$ & 12 & 13.2 & $12.1-14.2$ \\
\hline$B M I$ & 46 & 19.8 & $19.1-20.5$ & 13 & 21.1 & $19 . .5-22.7$ & 12 & 19.5 & $18.4-20.5$ \\
\hline$A M H$ & 36 & 4.8 & $3.8-5.9$ & 12 & 3.6 & $2.4-4.9$ & 11 & 4.0 & $2.4-5.5$ \\
\hline FSH Dose & 46 & 2276 & $2024-2560$ & 13 & 2700 & 1908-3492 & 12 & 2639 & $2392-2885$ \\
\hline Oocytes & 46 & 16.6 & $14.7-18.4^{*}$ & 13 & 11.3 & $8.3-14.3$ & 12 & 19.0 & $12.2-25.8^{* *}$ \\
\hline
\end{tabular}

${ }^{*} \mathrm{P}<0.05 ;{ }^{* *} \mathrm{P}<0.01$.

Here presented data, while in view of a relatively small data set, still to be considered as preliminary, nevertheless, warrant a careful reevaluation of current practice patterns, involving the use of $\mathrm{HCs}$ in association with IVF.

\section{Potential mechanism for findings}

As noted before, contraceptive progestins prevent ovulation through suppression of gonadotropin secretion and prevention of the LH surge [12,13]. Individual progestins, however, differ in their ability to suppress ovulation in animal models in the following declining order of potency: desogestrel > levonorgestrel > MPA > norgestimate $>$ norethindrone [35]. Drospirenone alone, and in combination with ethinyl estradiol, suppresses ovulation but does not completely suppress follicular development [36].

We demonstrate in this study that the two antiandrogenic progestins, drospirenone- and cyproterone acetate-containing OCs, led to similar oocyte numbers as in controls, who used no HCs at all, and, therefore, to significantly larger oocyte yields than in donor who utilized more androgenic HCs.

While the estrogen component may also have a role in ovarian suppression, significant increases in $\mathrm{AMH}$, antral follicle counts and ovarian volume values have been observed after discontinuation of a variety of $\mathrm{HCs}$, unrelated to the estrogen dose of HCs [37].

When using OCs, significant follicular growth occurs during the seven day pill-free interval, while continuous $\mathrm{OC}$ use results in greater suppression of follicular

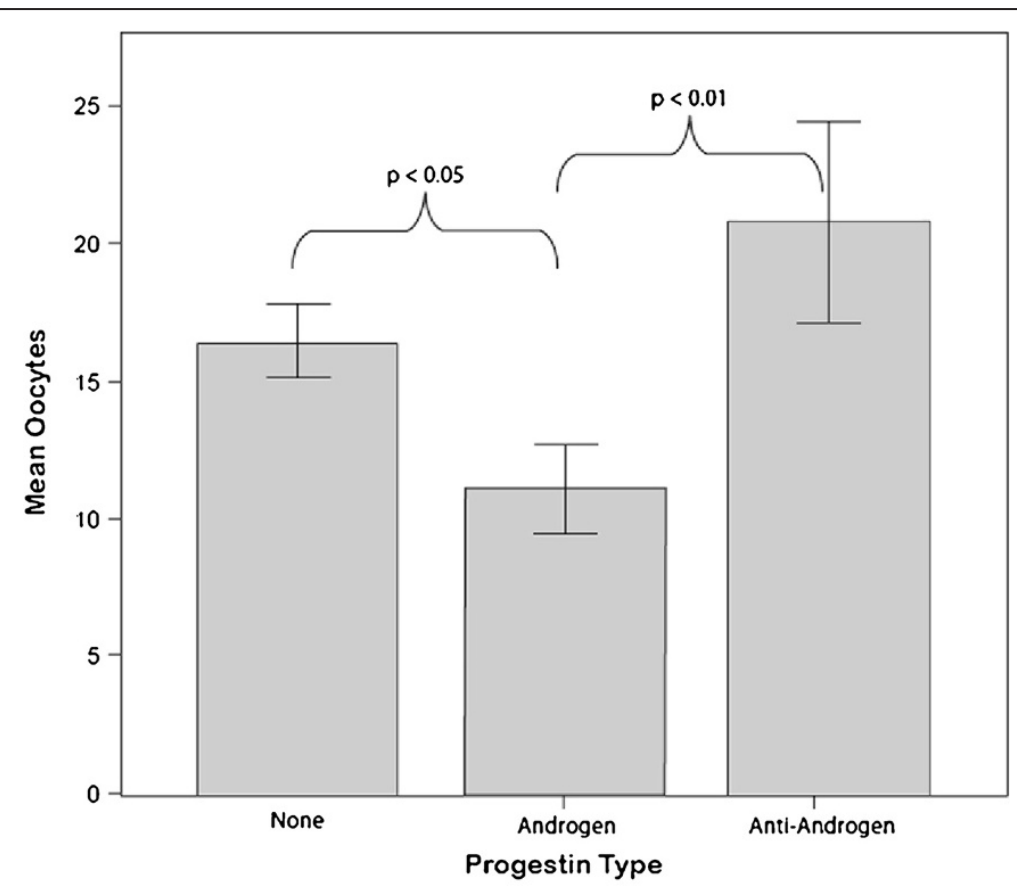

Error Bars +/- 1 SE

Figure 1 Oocyte yields of women using androgenic hormonal contraception. Oocyte yields of women using androgenic hormonal contraception were significantly lower than either women using no contraception or those using anti-androgenic contraceptives. 
activity $[38,39]$. This suggests that suppression of follicular growth in association with HCs may extend to antral and preantral stages of follicle maturation. Such a longterm effect may also be inferred from sometimes observed delays in resumption of normal cycles following cessation of HC. In such cases, ovarian biopsies demonstrate diffuse fibrous stroma, only primordial primary follicles and atrophic follicular cysts [40].

Considering that our group was the first to offer evidence that the weak androgen, dehydroepiandrosterone (DHEA) increases the growing follicle pool (i.e., functional ovarian reserve), resulting in higher oocyte yields and improved embryo quality [41-43], it may, on first glance, appear contradictory for this group of investigators to suggest that more androgenic progestins suppress antral follicle development. A closer look, however, reveals that such a contradiction may not really exist: Estrane and gonane progestin/ethinyl estradiol combination OCs suppress gonadotropins and, consequently, follicular development to a greater degree than drospirenoneand cyproterone acetate-containing OCs.

In early follicular development androgens work synergistically with FSH [44]. Therefore, utilizing a $\mathrm{HC}$, including an androgenic progestin, will inhibit gonadotropin support for the growing follicle but maintain androgen exposure. Such a constellation may negatively affect follicular development, leading to initial androgen-driven follicle growth but, in the end, to atresia of growing follicles because of lack of FSH support.

Assuming now a normal oocyte donor without ovarian PCO phenotype, supplemented with anti-androgenic HCs, she lacks both, the androgen driven growth of small growing follicles and the growth support from FSH. Very small follicles, therefore, will fail to grow, as in above described constellation, but will, therefore, also not reach stages of atresia. Assuming discontinuation of $\mathrm{HCs}$, and reconstitution of FSH support, these small follicles will, therefore, still have the ability to resume growth and development, leading to ultimately larger oocyte yields than with androgenic HCs.

Such a model of required synergism between androgens and FSH at small growing follicle stages to achieve normal follicle growth and maturation is well described in animal models [44]. It would suggest that FSH/androgen ratios at these early follicle growth stages may be predictive of later IVF cycle outcomes in humans.

\section{Limitations}

This study's principal limitation is the relative small number of study subjects, resulting in the description of this study as a pilot study in need of further validation. The potential importance of here first reported findings, however, warrant publication of these preliminary data to call attention to the likely suppressive effect of at least some HCs on ovarian reserve and encourage further investigations.

\section{Conclusions}

This study, therefore, in summary, suggests that HCs, containing progestins derived from androgenic estranes and gonanes, suppress functional ovarian reserve, most likely via gonadotropin suppression, which interrupts the normal synergism between androgens and FSH at small follicle growth stages, in turn impacting oocyte yields. Since many fertility centers routinely use OCs in preparation for IVF cycles, such a practice, even in young women with normal functional reserve, appears to have negative consequences on oocyte numbers, as here demonstrated in oocyte donors.

An even more profound negative impact from androgenic HCs can, however, be expected in women with diminished functional ovarian reserve. Further investigations are needed to determine whether they should be utilized at all. At minimum, the conclusion from this preliminary study should, however, be that androgenic HCs should, likely, be avoided in women with evidence of low functional ovarian reserve.

\section{Abbreviations}

AMH: Anti-Müllerian hormone; BMI: Body mass index; DHEA: Dehydroepiandrosterone; EE: Ethinyl estradiol; FSH: Follicle stimulationg hormone; HC: Hormonal contraceptives; hCG: Human chorionic gonadotropin; IU: International unit; IVF: In vitro fertilization; LH: Luteinizing hormone; OC: Oral contraceptives; OR: Ovarian reserve; PCO: Polycystic ovaries.

\section{Competing interests}

N.G, A.W. and D.H.B. have in the past received research support, speakers' honoraria and travel funds from various pharmaceutical and medical device companies, none, however, related to the subject of this paper. N.G. and D.H. $\mathrm{B}$, are listed as co-inventors of two awarded U.S. patents, claiming therapeutic benefits for DHEA, and potentially other androgens, in women with DOR. Both authors have other pending patent applications, regarding DHEA, and other androgens, and the FMR1 gene's effects on ovaries. N.G. owns shares in Fertility Nutraceuticals, LLC, a company that offers a DHEA product. N.G. and D.H.B. are receiving patent royalties from this company. N. $\mathrm{G}$. is also the owner of The CHR, where this research was conducted. Other authors have no conflicts to declare.

\section{Authors' contributions}

DHB and NG contributed equally to the manuscript, including study design, data analysis and writing of the manuscript, AK and DHB performed data analyses and statistical analyses, AW contributed to study design. All authors approved the final manuscript.

\section{Author details}

${ }^{1}$ Center for Human Reproduction, New York, NY, USA. ${ }^{2}$ Foundation for Reproductive Medicine, New York, NY, USA. ${ }^{3}$ Department of Gynecologic Endocrinology and Reproductive Medicine, Medical University Vienna, Vienna, Austria.

Received: 31 January 2013 Accepted: 13 March 2013

Published: 4 April 2013

\section{References}

1. Ferraretti AP, La Marca A, Fauser BC, Tarlatzis B, Nargund G, Gianaroli L: ESHRE consensus on the definition of 'poor response' to ovarian 
stimulation for in vitro fertilization: the Bologna criteria. Hum Reprod 2011, 26:1616-1624.

2. Surrey ES: Management of the poor responder: the role of $\mathrm{GnRH}$ agonists and antagonists. J Assist Reprod Genet 2007, 24:613-619.

3. Lindheim SR, Barad DH, Witt B, Ditkoff E, Sauer MV: Short-term gonadotropin suppression with oral contraceptives benefits poor responders prior to controlled ovarian hyperstimulation. J Assist Reprod Genet 1996, 13:745-747.

4. Fisch B, Royburt M, Pinkas H, Avrech OM, Goldman GA, Bar J, Tadir Y, Ovadia J: Augmentation of low ovarian response to superovulation before in vitro fertilization following priming with contraceptive pills. Isr J Med Sci 1996, 32:1172-1176.

5. Biljan MM, Mahutte NG, Dean N, Hemmings R, Bissonnette F, Tan SL: Pretreatment with an oral contraceptive is effective in reducing the incidence of functional ovarian cyst formation during pituitary suppression by gonadotropin-releasing hormone analogues. J Assist Reprod Genet 1998, 15:599-604.

6. Patton PE, Burry KA, Wolf DP, Kiessling AA, Craemer MJ: The use of oral contraceptives to regulate oocyte retrieval. Fertil Steril 1988, 49:716-718.

7. Kemeter $\mathrm{P}$, Feichtinger $\mathrm{W}$ : A fixed stimulation protocol for in vitro fertilization (IVF) without determinations of hormones in blood. Acta Eur Fertil 1989, 20:63-70.

8. Meldrum DR, Scott RT Jr, Levy MJ, Alper MM, Noyes N: Oral contraceptive pretreatment in women undergoing controlled ovarian stimulation in ganirelix acetate cycles may, for a subset of patients, be associated with low serum luteinizing hormone levels, reduced ovarian response to gonadotropins, and early pregnancy loss. Fertil Steril 2009, 91:1963-1965.

9. Duvan $\mathrm{Cl}$, Berker B, Turhan NO, Satiroglu H: Oral contraceptive pretreatment does not improve outcome in microdose gonadotrophin-releasing hormone agonist protocol among poor responder intracytoplasmic sperm injection patients. J Assist Reprod Genet 2008, 25:89-93.

10. Kovacs P, Barg PE, Witt BR: Hypothalamic-pituitary suppression with oral contraceptive pills does not improve outcome in poor responder patients undergoing in vitro fertilization-embryo transfer cycles. $J$ Assist Reprod Genet 2001, 18:391-394.

11. Smulders B, van Oirschot SM, Farquhar C, Rombauts L, Kremer JA: Oral contraceptive pill, progestogen or estrogen pre-treatment for ovarian stimulation protocols for women undergoing assisted reproductive techniques. Cochrane Database Syst Rev 2010, 11:CD006109.

12. Mishell DR: The effect of contraceptive steroids on hypothalamicpituitary function. Am J Obstet Gynecol 1977, 128:60-74.

13. Dericks-Tan JS: Influence of oral contraceptives on integrated secretion of gonadotropins. Contracept 1992, 46:369-377.

14. Sitruk-Ware $R$, Nath $A$ : The use of newer progestins for contraception. Contracept 2010, 82:410-417.

15. Fang $H$, Tong W, Branham WS, Moland CL, Dial SL, Hong H, Xie Q, Perkins R, Owens W, Sheehan DM: Study of 202 natural, synthetic, and environmental chemicals for binding to the androgen receptor. Chem Res Toxicol 2003, 16:1338-1358.

16. Sitruk-Ware R: Pharmacological profile of progestins. Maturitas 2008, 61:151-157.

17. Africander D, Verhoog N, Hapgood JP: Molecular mechanisms of steroid receptor-mediated actions by synthetic progestins used in HRT and contraception. Steroids 2011, 76:636-652.

18. Elger W, Beier S, Pollow K, Garfield R, Shi SQ, Hillisch A: Conception and pharmacodynamic profile of drospirenone. Steroids 2003, 68:891-905.

19. Winneker RC, Bitran D, Zhang Z: The preclinical biology of a new potent and selective progestin: trimegestone. Steroids 2003, 68:915-920.

20. Schindler AE, Campagnoli C, Druckmann R, Huber J, Pasqualini JR, Schweppe KW, Thijssen JH: Classification and pharmacology of progestins. Maturitas 2008, 61:171-180.

21. Ndefo UA, Mosely N: Estradiol valerate and estradiol valerate/dienogest (natazia) tablets: the first four-phasic oral contraceptive. Pharm Therapeutics 2010, 35:61461-61467.

22. de Vet AR: Antimüllerian hormone serum levels: a putative marker for ovarian aging. Fertil Steril 2002, 77:357-362.

23. Singer $\mathrm{T}$, Barad DH, Weghofer $\mathrm{A}$, Gleicher $\mathrm{N}$ : Correlation of antimullerian hormone and baseline follicle-stimulating hormone levels. Fertil Steril 2009, 91:2616-2619.

24. Gleicher N, Weghofer A, Barad DH: Discordances between follicle stimulating hormone (FSH) and anti-Mullerian hormone (AMH) in female infertility. Reprod Biol Endocrinol 2010, 8:64.
25. Gleicher N, Weghofer A, Barad DH: Anti-Mullerian hormone (AMH) defines, independent of age, low versus good live-birth chances in women with severely diminished ovarian reserve. Fertil Steril 2010, 94:2824-2827.

26. Gleicher N, Ryan E, Weghofer A, Blanco-Mejia S, Barad DH: Miscarriage rates after dehydroepiandrosterone (DHEA) supplementation in women with diminished ovarian reserve: a case control study. Reprod Biol Endocrinol 2009, 7:108.

27. Somunkiran AR: Anti-Müllerian hormone levels during hormonal contraception in women with polycystic ovary syndrome. Eur J Obstet Gynecol Reprod Biol 2007, 134:196-201

28. Venturoli S, Ravaioli B, Bagnoli A, Colombo FM, Macrelli S, ladarola I, Vianello F, Mancini F, Flamigni C: Contraceptive and therapeutic effectiveness of two low-dose ethinylestradiol and cyproterone acetate regimens in the treatment of hirsute patients. Eur J Contracept Reprod Health Care 1998, 3:29-33.

29. De Leo V, Lanzetta D, Morgante G, De Palma P, D'Antona D: Inhibition of ovulation with transdermal estradiol and oral progestogens in perimenopausal women. Contracept 1997, 55:239-243.

30. Arbo E, Vetori DV, Jimenez MF, Freitas FM, Lemos N, Cunha-Filho JS: Serum anti-mullerian hormone levels and follicular cohort characteristics after pituitary suppression in the late luteal phase with oral contraceptive pills. Hum Reprod 2007, 22:3192-3196.

31. Kerkhof GF, Leunissen RW, Willemsen RH, de Jong FH, Visser JA, Laven JS, Hokken-Koelega AC: Influence of preterm birth and small birth size on serum anti-Mullerian hormone levels in young adult women. Eur J Endocrinol 2010, 163:937-944

32. Streuli I, Fraisse T, Pillet C, Ibecheole V, Bischof P, de Ziegler D: Serum antimullerian hormone levels remain stable throughout the menstrual cycle and after oral or vaginal administration of synthetic sex steroids. Fertil Steril 2008, 90:395-400.

33. Steiner AZ, Stanczyk FZ, Patel S, Edelman A: Antimullerian hormone and obesity: insights in oral contraceptive users. Contracept 2010, 81:245-248.

34. Petrie KA, Torgal AH, Westhoff CL: Matched-pairs analysis of ovarian suppression during oral vs. vaginal hormonal contraceptive use. Contracept 2011, 84:e1-e4

35. Phillips A, Hahn DW, Klimek S, McGuire JL: A comparison of the potencies and activities of progestogens used in contraceptives. Contracept 1987, 36:181-192.

36. Rosenbaum P, Schmidt W, Helmerhorst FM, Wuttke W, Rossmanith W, Freundl F, Thomas K, Grillo M, Wolf A, Heithecker R: Inhibition of ovulation by a novel progestogen (drospirenone) alone or in combination with ethinylestradiol. Eur J Contracept Reprod Health Care 2000, 5:16-24.

37. van den Berg MH, van Dulmen-den BE, Overbeek A, Twisk JW, Schats R, van Leeuwen FE, Kaspers GJ, Lambalk CB: Comparison of ovarian function markers in users of hormonal contraceptives during the hormone-free interval and subsequent natural early follicular phases. Hum Reprod 2010, 25:1520-1527.

38. Schlaff WD: Manipulation of the pill-free interval in oral contraceptive pill users: the effect on follicular suppression. Am J Obstet Gynecol 2004, 190:943-9451.

39. Baerwald AR: Ovarian follicular development is initiated during the hormone-free interval of oral contraceptive use. Contracept 2004, 70:371-377

40. Said S, el Habashy MA, Osman MM, Shams AT, Madwar AY, Nayel SA Ovarian morphology and histopathology in post pill amenorrhea. Asia Oceania J Obstet Gynaecol 1987, 13:15-19.

41. Barad D, Brill H, Gleicher N: Update on the use of dehydroepiandrosterone supplementation among women with diminished ovarian function. J Assist Reprod Genet 2007, 24:629-634.

42. Barad D, Gleicher N: Effect of dehydroepiandrosterone on oocyte and embryo yields, embryo grade and cell number in IVF. Hum Reprod 2006, 21:2845-2849

43. Barad DH, Gleicher N: Increased oocyte production after treatment with dehydroepiandrosterone. Fertil Steril 2005, 84:756.

44. Gleicher N, Weghofer A, Barad DH: The role of androgens in follicle maturation and ovulation induction: friend or foe of infertility treatment? Reprod Biol Endocrinol 2011, 9:116.

doi:10.1186/1477-7827-11-28

Cite this article as: Barad et al:: Does hormonal contraception prior to in vitro fertilization (IVF) negatively affect oocyte yields? - A pilot study. Reproductive Biology and Endocrinology 2013 11:28. 